Document downloaded from:

http://hdl.handle.net/10251/136400

This paper must be cited as:

Antonino-Daviu, JA.; Quijano-Lopez, A.; Rubbiolo, M.; Climente Alarcon, V. (2018). Advanced analysis of motor currents for the diagnosis of the rotor condition in electric motors operating in mining facilities. IEEE Transactions on Industry Applications. 54(4):3934-3942. https://doi.org/10.1109/TIA.2018.2818671

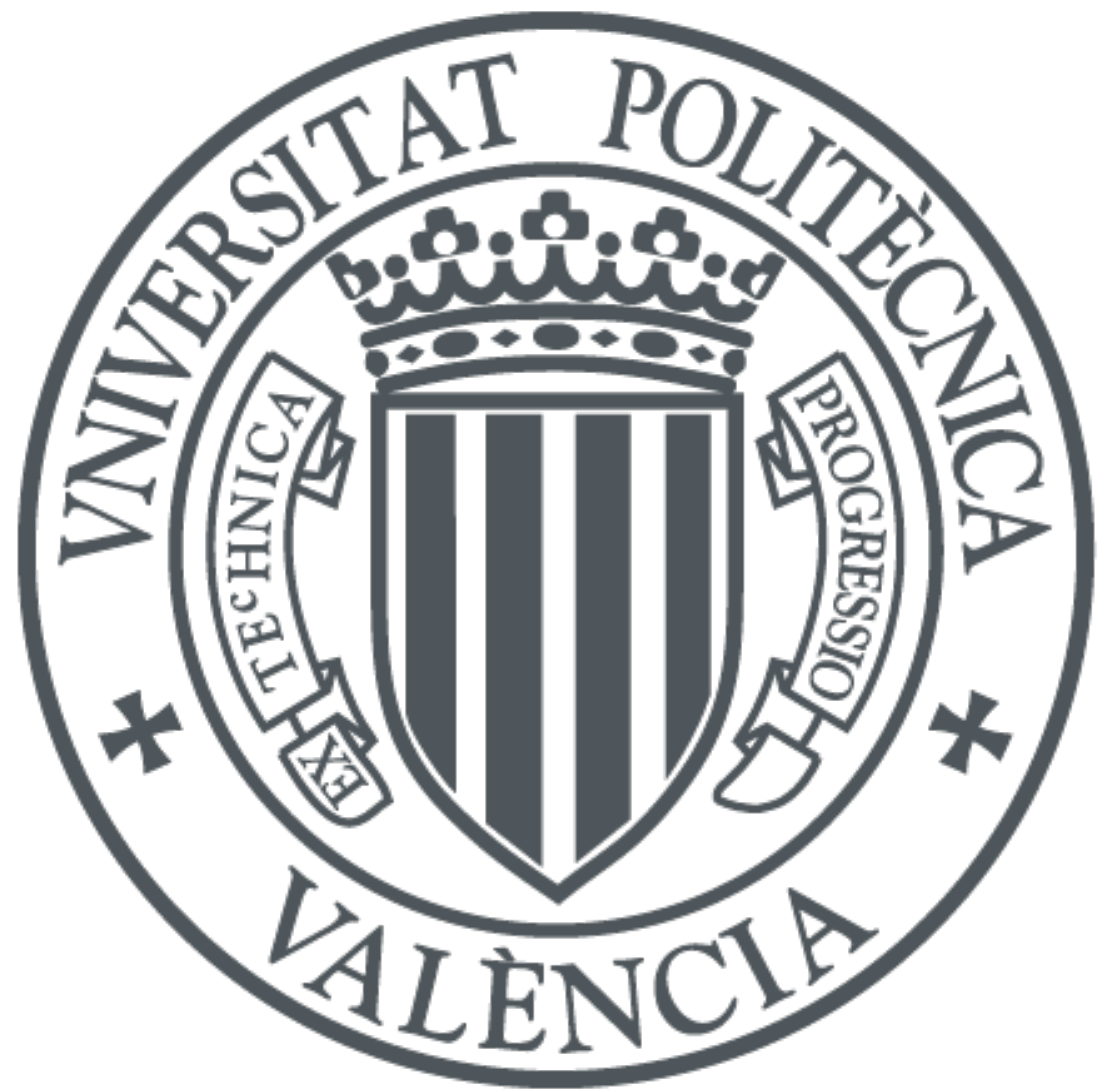

The final publication is available at

https://doi.org/10.1109/TIA.2018.2818671

Copyright Institute of Electrical and Electronics Engineers

Additional Information 


\section{Advanced analysis of motor currents for the diagnosis of the rotor condition in electric motors operating in mining facilities}

\author{
Jose Antonino-Daviu, \\ Senior Member, IEEE \\ Alfredo Quijano López
}

Universitat Politecnica de Valencia

Instituto Tecnológico de la Energía

Camino de Vera s/n, 46022, Valencia, SPAIN; joanda@die.upv.es

\author{
Martin Rubbiolo \\ Motors and Generators Inc. \\ 315 South 40th St. \\ Boulder, CO, USA \\ info@motoresygeneradores.com
}

\author{
Vicente Climente-Alarcon \\ Member IEEE \\ Aalto University \\ Dep. Elec. Engineering and Automation \\ P. O. Box 15500 \\ FI-00076 Aalto, FINLAND \\ viclial@ieee.org
}

\begin{abstract}
Predictive maintenance of electric motors is a topic of increasing importance in many industrial applications. The mining industry is not an exception; many electric motors operating in mining facilities are critical machines and their unexpected failures may imply significant losses and can be hazardous for the users. Due to these facts, an increasing research effort has been dedicated to investigate on new techniques that are able to provide a reliable diagnostic of the motor condition. Over recent years, monitoring of electrical quantities (e.g. motor currents) has emerged as a very attractive option for determining the health of several motor parts (rotor, eccentricities, bearings) due to its very interesting advantages: possibility of remote motor monitoring, non-invasive nature, simple application, broad fault coverage... The traditional methods based on analysis of motor currents during steady-state operation (MCSA) are being complemented, when not replaced, by more reliable approaches. This work applies an innovative transient based methodology (ATCSA) to several case studies referred to large motors operating in mining facilities. The results prove how this modern methodology enables to overcome some important drawbacks of the classical MCSA, such as its unsuitability under varying speed conditions, and may provide an earlier indication of rotor electrical asymmetries under such working conditions.
\end{abstract}

Index Terms-Induction motors; mining; fault diagnosis; transient analysis; rotor; reliability; fault detection; wavelet.

\section{INTRODUCTION}

There are more than 300 million electric motors in the world. These machines intervene in a countless number of industrial applications and they are considered as the 'workhorses' of modern societies [1]. The mining industry is not an exception; electric motors are critical assets in mining facilities where they drive a diversity of loads. One particularity of the motors in mining applications is their variable operation regimes. Unlike the motors in other industries (such as petrochemical), which usually work under rather stable regimes, most of the motors involved in mining processes are subjected to frequent transients and often work under intermittent duty cycles or cycles with variable loads (frequent starts, decelerations, stops, load changes...).

The most widespread electric motors are the cage induction motors. In comparison with other alternatives (synchronous machines, wound rotor asynchronous motors, D.C. motors...) cage motors have important advantages, such as their robustness, simplicity and lower cost. However, this does not imply that they are not prone to suffer faults: bearing failures, stator insulation faults, rotor damages and core failures are the most common types of faults in induction motors [1-3].

Rotor damages are particularly dangerous. Since the occurrence rate of rotor faults is, in relative terms, much lower than those of other types of failures (bearings, insulation), many users have the erroneous belief that the rotor never fails or that it does not require a proper maintenance. Although rotor damages do not usually lead to catastrophic effects, many industrial cases of forced motor outages caused by the detachment of fragments of broken rotor bars or by the protrusion of broken bars that led to the damage of the stator insulation have been recently reported [2]. But, even if the rotor fault does not cause immediate catastrophic failure, the damage progressively propagates, increasing in severity and affecting the motor efficiency. If the fault severity gets worse, it can even impede the motor startup, causing unplanned production shutdowns. Moreover, rotor faults are much more frequent in large motors starting under high inertias and operating under intermittent cycles that include frequent startups or abrupt load variations. These situations are frequent in the mining industry and this is the reason why the detection of rotor faults is especially relevant in this industry. Fig. 1 shows a picture of a rotor fault in a large motor operating in a mining industry.

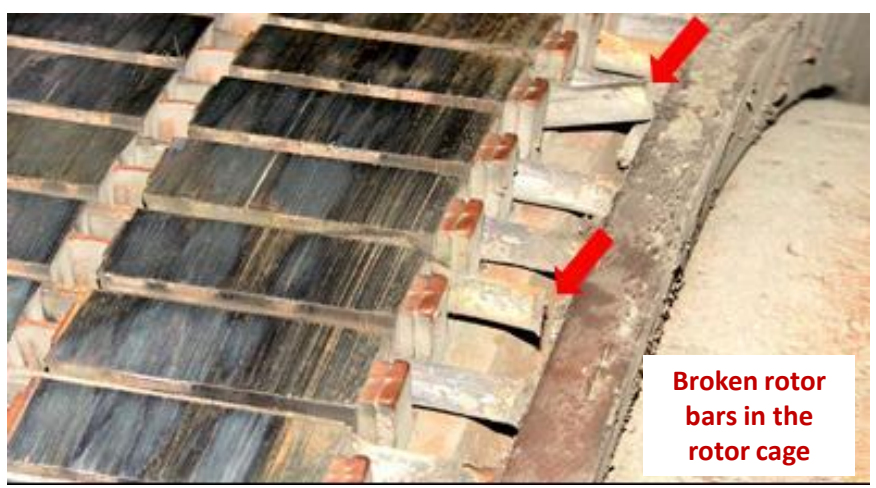

Fig.1 Example of rotor fault in a $320 \mathrm{~kW}$ motor operating in a mining facility. 
The classical method for the assessment of the rotor condition in induction motors is the well-known Motor Current Signature Analysis (MCSA) [1, 4-5] that relies on capturing the waveform of the current demanded by the motor during steady-state operation, analyzing it with the the Fast Fourier Transform (FFT) and evaluating the amplitude of certain components or harmonics that are amplified by the rotor fault. This approach has given good results for a certain range of industrial applications, where the operation regime is rather stable. However, it has been proven its unsuitability in applications where the motor speed is continuously changing (due to frequent transients, load variations, supply modifications...), which are rather common in the mining industry, as it was preliminarily proven in [6]. Moreover, in the recent decade, it has been shown that in some industrial situations the application of MCSA may cause erroneous diagnostics (false indications), which can lead to severe consequences for the corresponding industry [7-11].

Due to the aforementioned drawbacks of MCSA, a new methodology has been introduced over these last few years. Unlike the classical MCSA, this new trend (known as Advanced Transient Current Signature Analysis, ATCSA [69]) relies on the analysis of the motor current regardless of its operation regime, so it includes the analysis of currents during transient operation. In its simplest and most studied modality, this new approach is based on the analysis of the current demanded by the motor during its connection process (startup current) [8]. The analysis of this signal has been recently proven to be a very powerful informational source to diagnose the condition of the rotor, overcoming most of the MCSA drawbacks. For instance, it has been proven its potential, even under variable regimes [6], as well as its reliability in situations where MCSA may provide false indications [7-11]. These advantages are extremely helpful in the mining industry, in cases where, as it will be shown in this paper, ATCSA can help to provide a very reliable diagnosis while MCSA may not be helpful due to the variable operation of these motors.

The present paper presents, for the first time, the combined application of advanced signal processing tools adapted to transient analysis (ATCSA) to the detection of electrical rotor asymmetries in real mining field cases in which the MCSA method was not reliable. The cases are referred to motors that operate in mining facilities in South America and Central Europe [14] and were suggested by the own manufacturers of well-known condition monitoring devices. This work combines the application of sophisticated continuous transforms for obtaining the characteristic patterns created by the fault components, with discrete transforms to compute the fault severity indicators. The results prove the reliability of this advanced methodology for diagnosing the rotor condition, even in especially adverse situations, such as motors operating under variable speed regimes. The importance of ATCSA is further highlighted with a simulated result showing that under severe transients, usual in the mining industry, incipient defects in the secondary circuit of an induction motor may increase its effect and hence provide better means for early diagnosis.

\section{CLASSICAL METHOD BASED ON CURRENT ANALYSIS (MCSA) VS NEW TRANSIENT-BASED APPROACH (ATCSA)}

In this section, the two main approaches based on current analysis for rotor condition assessment in induction motors are compared: on the one hand, the classical method based on the FFT of the steady-state current (MCSA) and, on the other hand, the new proposed approach relying on analysis of the startup current (ATCSA). The foundations of each are described and the advantages of ATCSA are exposed.

\section{A. Classical method: MCSA}

As commented above, the classical method for rotor condition monitoring in induction motors is the well-known MCSA. Its application includes three different steps [1, 4-5]: 1) capture of the waveform of the current demanded by the motor during steady-state, 2) performing the FFT analysis of the steady-state current signal and 3) evaluation of the amplitudes of the harmonics amplified by rotor damages.

When rotor damages (broken or partially broken rotor bars, cracked end rings and aluminum injection voids in aluminum cages) are present, two main harmonic families are amplified [1, 15]. These families are given by (1) and (2), where $f$ is the supply frequency, $s$ is the slip, $k=$ natural number in (1) and $k / p=1,3,5 \ldots$ in (2) [1, 15]. Among them, the most relevant components, which are usually employed to assess the level of rotor damage are sideband harmonics that are obtained from (1) for $k=1$. The few commercial devices that can perform the assessment of the rotor condition based on current analysis rely on the evaluation of the amplitudes of these harmonics and, more specifically, on the amplitude of the lower sideband harmonic (LSH) - the one obtained for negative sign and $k=1$ in (1). Amplitudes of the LSH between $-55 \mathrm{~dB}$ and $-45 \mathrm{~dB}$ relative to that of the fundamental are usually indicative of the presence of rotor damage [2].

Sometimes, the identification of other harmonics given by (1) and/or (2) are also employed as complementary informational sources for the diagnosis, especially when the identification of the sideband harmonics is not easy. In fact, it is rather usual the use of the harmonics given by $f \cdot(5-4 \cdot s)$ and $f \cdot(5-6 \cdot s)$, which are obtained from (2) for $k / p=5$ [16]. The detection of these harmonics is a complementary evidence of the presence of the rotor failure. To illustrate the application of MCSA, Fig.2 shows the identification of the sideband harmonics and the $f \cdot(5-4 \cdot s)$ and $f \cdot(5-6 \cdot s)$ components in a motor with two broken bars (out of 28). The clear conclusion is that the fault is present in the machine.

$$
\begin{aligned}
f_{b b 1} & =[1 \pm 2 k s] f \\
f_{b b 2} & =\left[\frac{k}{p}(1-s) \pm s\right] \cdot f
\end{aligned}
$$

MCSA has provided satisfactory results when diagnosing rotor faults as well as other electromechanical failures (eccentricities and even bearing damages) in a broad range of 
motors operating under diverse conditions. However, it also has some important constraints that have been extensively reported in recent works. These can be summarized in two main points [12]:

1) MCSA is not suitable application under variable speed or supply conditions: if the motor speed changes during the capture of the current, the slip $s$ will also do. The immediate consequence is that the slip-dependent fault harmonics, given by (1) and (2) will also change. The result is that a certain component will not appear as single 'peak' in a specific frequency of the spectrum, but it will spread over a certain frequency range. Hence, it will be very difficult to evaluate the real amplitude of a certain fault component since it will be distributed over different frequencies. This constraint is especially critical in some mining applications where the speed continuously changes, as it will be shown in this paper [6]. Analogously, if the supply conditions change during the capture, the effect is even worse: if the supply frequency varies, the frequencies of fault components will also change (see (1) and (2)), but also will do the fundamental component. This situation is common in inverter-fed motors, where the converter control loop changes the supply frequency depending on the motor loading conditions.
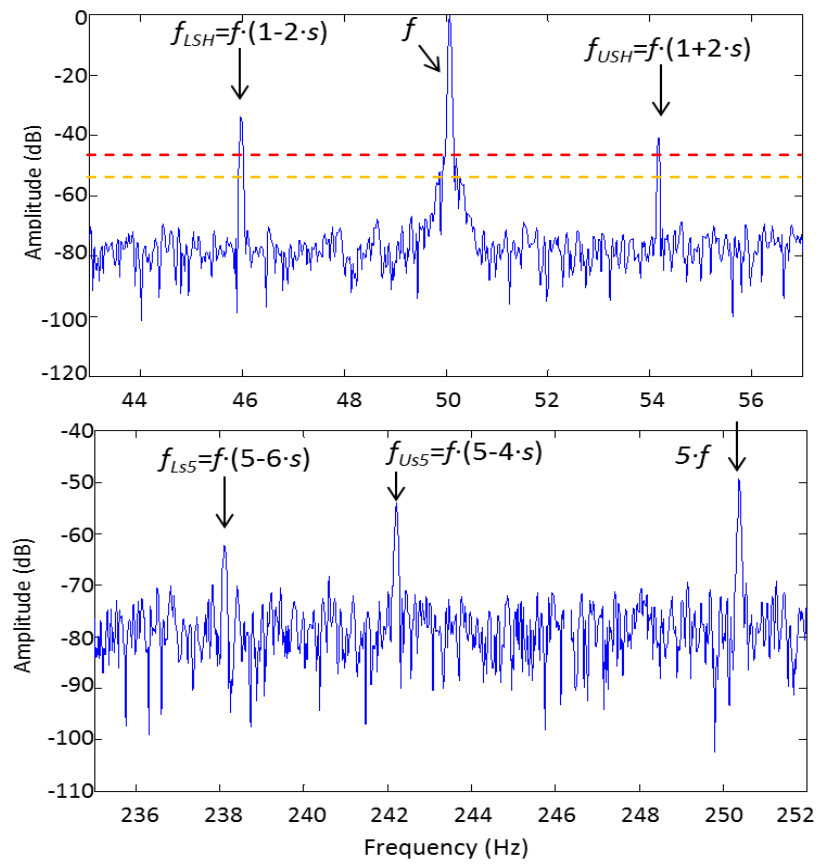

Fig. 2 MCSA of a motor with two broken rotor bars: sideband components (top) and fault components at $f \cdot(5-4 \cdot s)$ and $f \cdot(5-6 \cdot s)$ (bottom).

2) MCSA may provide occasional false indications: It has been extensively reported over recent years that there are some industrial cases where the use of MCSA can lead to erroneous diagnostics. These wrong diagnostics can be classified into two categories: false positive indications (i.e. diagnosing the rotor as faulty when it is healthy) and false negative indications (i.e. diagnosing the rotor as healthy when its actual condition is faulty). There are a number of situations that can lead to these false indications and they have been reported in detail in recent works [7-11, 17-19]. These situations are related either to operational regimes of the machine (diagnosis under no load conditions, presence of load fluctuations, etc...) or to its constructive characteristics (existence of cooling axial ducts, rotor core anisotropy issues, broken outer bars in double cage rotors, etc...). Luckily, these situations are much less frequent than those where MCSA provides correct results. However, the consequences of eventual false negative or positive indications can be very negative, especially in terms for economic losses [2, 7-11]. This makes the use of complementary tools to ratify the presence of the aforementioned faults advisable.

\section{B. New transient-based methodology: ATCSA}

In order to overcome the MCSA drawbacks, a novel approach for fault diagnosis in electric motors has been recently proposed. This new approach is based on the analysis of the current regardless of the operation regime of the motor, so it includes the analysis of currents during transient motor operation (ATCSA). The simplest and most extended modality of this approach relies on the analysis of the startup current [8]. Unlike the MCSA method, where the objective is to identify frequency components in the FFT spectrum, in ATCSA the goal is to track the evolutions of fault components during the startup. The underlying idea is simple: since the frequencies of the fault components, given by (1) and (2), are functions on the slip $s$, they will vary as the slip does. Since, during a direct-on-line startup the slip $s$ changes between 1 and near 0 , the fault components' frequencies will change accordingly. For instance, according to the expression $f \cdot(1-2 \cdot s)$, the frequency of the LSH will drop during the startup from $f$ (when $s=1$ ) to $0 \mathrm{~Hz}$ (when $s=1 / 2$ ) and will increase again to near $f \mathrm{~Hz}$ (when $\mathrm{s} \approx 0$ at steady-state) [8]; hence if this frequency evolution is plotted over time, the LSH will follow a very characteristic $\mathrm{V}$ pattern that is a reliable evidence of the presence of the rotor failure (see Fig. 3 (b)). The problem is that, in order to detect this type of evolutions (a frequency change in time) is necessary to employ proper signal processing tools able to provide a timefrequency representation of the analyzed signal. These tools are known as time-frequency (T-F) transforms. There are many available T-F tools; in past works, they were classified in discrete transforms and continuous transforms, each group having its own advantages and drawbacks $[13,20]$.

In this particular work, two different T-F tools are used: on the one hand, an optimized version of the Short Time Fourier Transform (STFT) is employed for the continuous analyses. The STFT is based on the following idea [21]: the analyzed signal is multiplied by a window function centered at $t$ that is different from zero only for a short time period [22]. The expression of this transform is given by $(3) ; h(\tau)$ is the window function (often a Hann window or a Gaussian window although other functions can be also used), while $x(\tau)$ is the analyzed signal. Then, the Fourier transform of the 
resulting signal is obtained, while moving the window function along the time axis, yielding a two-dimensional representation of the signal (see (4)). In such way, a set of spectra are obtained; the total set is known as $\operatorname{PSP}(t, w)$, which is a time-frequency representation of the analyzed signal (known as spectrogram) [21].

$$
\begin{gathered}
x_{t}(\tau)=\frac{1}{\sqrt{2 \cdot \pi}} \cdot \int_{-\infty}^{\infty} x(\tau) \cdot h(\tau-t) d \tau \\
X_{t}(\omega)=\frac{1}{\sqrt{2 \cdot \pi}} \cdot \int_{-\infty}^{\infty} x(\tau) \cdot h(\tau-t) e^{-j \omega t} \cdot d \tau
\end{gathered}
$$

On the other hand, a discrete time-frequency tool, the Discrete Wavelet Transform (DWT) is employed for the computation of the fault severity indicator. When the DWT is applied to a certain sampled signal $i(t)$, this signal is decomposed into a set of signals (wavelet signals): an approximation signal at a certain decomposition level $n\left(a_{n}\right)$ plus $n$ detail signals ( $d_{j}$ with $j$ varying from 1 to $n$ ) [23]. Each wavelet signal (approximation and detail) has an associated frequency band, the limits of which are wellestablished, once the sampling rate $\left(f_{s}\right)$ of the original analyzed signal is known, in accordance with an algorithm enunciated by S. Mallat (Subband Coding Algorithm) [24].

The fault severity indicator based on the DWT was proposed in previous works $\left(\gamma_{D 7}\right)[8,10]$ and it is defined as the ratio between the energy of a specific wavelet signal (usually $d_{7}$ ) and that of the total startup current signal. The expression of this indicator is given by (5), where $i_{j}$ is the value of the $j$ th sample of the current signal; $d_{7}(j)$ is the $j$ element of the wavelet signal $d_{7} ; N_{b}$ is the number of the sample corresponding to the starting point of the second group of oscillations in $d_{7}$ (return trip of LSH); $N_{s}$ is the number of sample at which these oscillations are extinguished $[8,10]$. According to the experience of the authors, values of $\gamma_{D 7}$ lower than $50 \mathrm{~dB}$ are indicative of rotor damage.

$$
\gamma_{D 7}(d B)=10 \cdot \log \left[\frac{\sum_{j=N b}^{N s} i_{j}^{2}}{\sum_{j=N b}^{N s}\left[d_{7}(j)\right]^{2}}\right]
$$

One of the advantages of ATCSA is that it does not only rely on the detection of the LSH evolution for detecting rotor damages. Each fault harmonic given by (1) or (2) has its own evolution as the slip changes and, hence, it is possible to detect the evolutions of all these harmonics with the T-F transforms, increasing the reliability in the diagnostic [13]. For instance, the USH, given by $f \cdot(1+2 \cdot s)$ will evolve between $3 \cdot f$ (when $s=1$ ) and $f$ (when $s \approx 0$ at steady-state). Fig. 3 illustrates the application of ATCSA and shows the clear differences between a healthy motor and a motor with faulty rotor. In this figure, the STFT is used and it clearly shows the aforementioned V-shaped evolution for the faulty case.
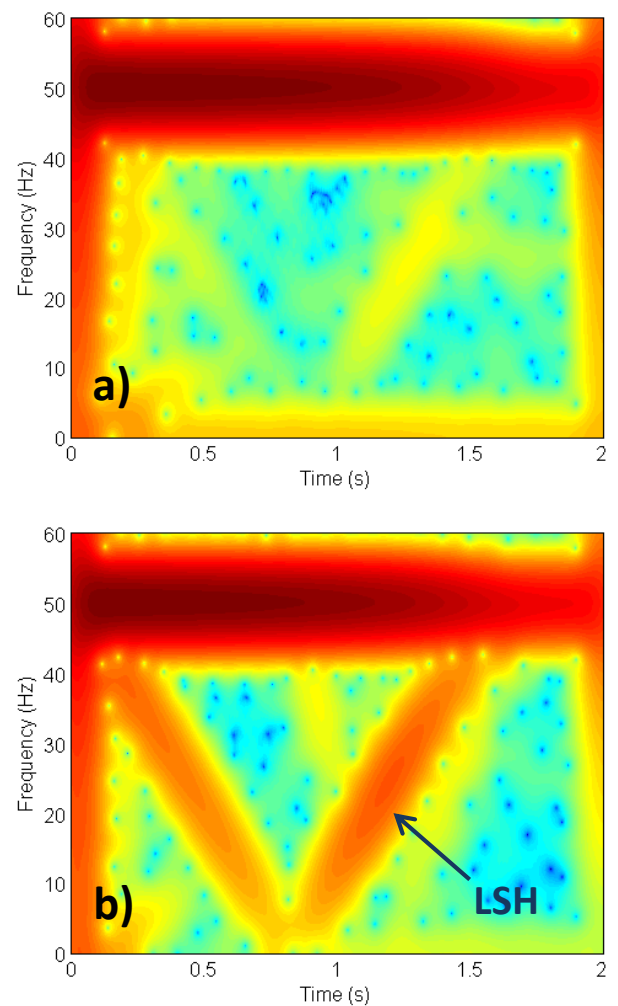

Fig. 3 Application of ATCSA to the startup current of a motor with: (a) healthy rotor and (b) rotor with two broken bars.

Note that ATCSA brings important advantages: firstly, for detection purposes, the identification of patterns is much more reliable than identifying a single component in a frequency spectrum, avoiding false indications of classical MCSA. In addition, ATCSA is valid for any operation regime of the motor. Secondly, heavy transients, usual in the mining industry, load the rotor cage with currents more than six times higher than the ones existing during operation at rated speed thus, increasing the electrical asymmetry of the cage due to differential heating when incipient faults are present. In order to provide an example of this effect, the model presented in [25] has been used to simulate for a long startup transient the rotor quantities in two damaged rotors, one having one of the bar's endings reduced to $12 \%$ of its original cross-section and other $8 \%$, thus limiting the current that flows into the end ring. The variation of resistance of the damaged bars during the transient compared to a healthy one is depicted in Fig. 4. At 5 seconds, when the slip has been reduced to $s=0.084$, the bar with the highest level of damage (8\%) exhibits an increase in resistance of $38 \%$ compared to the $29 \%$ at the beginning of the transient ( $s=1$, cold state). For the other case (12\% of remaining cross section at one of the bar's end) the rise in resistance is $23 \%$ compared to $17 \%$ at connection. These differences are reduced as the rotor further approaches synchronous speed -still during the transient- and the values of the currents decrease. Therefore, the careful quantification 
of the energy present in the evolution of the LSH in the timefrequency diagram, especially the ascending branch, can yield a more accurate view of the state of the cage compared to stationary analysis methods.

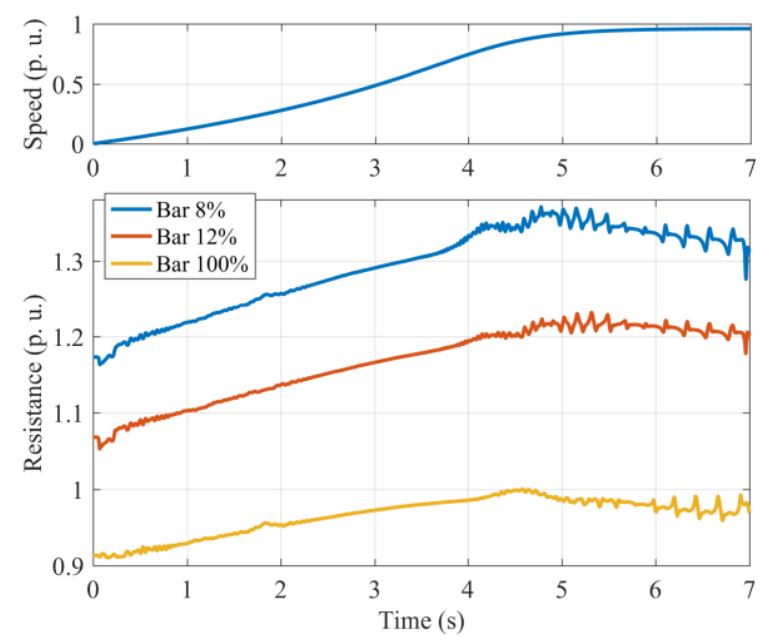

Fig. 4. Rotor speed during a direct-on-line startup (top) and smoothed evolution of the rotor bars' resistance for two damaged cages (bottom). The differential heating further increases the values for the damaged ones compared to the healthy as the transient progresses.

\section{FIELD CASES IN REAL MINING FACILITIES}

This section presents several cases corresponding to field motors operating in different mining facilities in South America and Central Europe. These were all large machines and most of them were operating under variable duty cycles, a fact that made, in some of the analyzed cases, the application of the classical MCSA difficult.

\section{A. H.V. motors in giant bucket wheel excavators}

The first analyzed case is related to a set of four motors that belong to giant bucket wheel stone excavators (commonly known as 'palas Komatsu'), which were operating in open mining facilities in South America [6]. All analyzed motors were 4-pole machines which were rated 7.2 $\mathrm{kV}$ and $1450 \mathrm{~kW}$. The particularity of these motors was relying on their variable operating conditions, since their load (and, hence, the slip) did not remain constant but was varying all the time.

The particular operation of these motors caused that the application of MCSA was unsuitable: when the load (slip) changes during the capture of the current, the fault frequencies (that depend on the slip, as shown in (1) and (2)) also change and, consequently, they do not fall at a specific frequency value but they spread within a certain frequency range in the FFT spectrum. This makes the evaluation of the real amplitudes of the fault harmonics and, subsequently, the evaluation of the rotor condition via MCSA very difficult.

This unsuitability of MCSA for assessing the rotor condition in this field case was illustrated by the fact that, when using a commercial device relying on MCSA for rotor condition monitoring, the diagnostics provided were erratic and even erroneous in some cases, due to the varying nature of the load: if the slip remained approximately constant during a capture, the fault harmonic amplitude was able to be properly determined. Otherwise, the value of the fault component amplitude, computed by the device, was erroneous since it spread through different spectral frequencies.

Moreover, in some of the analyzed cases the oscillating nature of the driven load was introducing harmonics very similar to those caused by the fault, leading to false positive indications, as it was already reported in previous works [8, 17]. All these problems can be satisfactorily solved if the novel startup analysis approach (ATCSA) was applied.

Fig. 5 shows, for each analyzed motor (M1 to M4), the evolution of the LSH amplitude relative to the fundamental for different successive measurements that were carried out in the corresponding motor (the alarm region is between the red and orange dotted lines and below the red line we would have a clear faulty condition). A first overview shows the significant variation of the LSH amplitude for each machine. This is caused by the aforementioned variation of the motor load that was influencing the evaluated amplitude of that harmonic. On the other hand, Fig. 6 shows the ATCSA analyses of the startup current for the four considered motors. A more detailed study of Figs.5 and 6, for each specific motor, leads to the following conclusions:

- With regards to motor M1, the value of the LSH amplitude at successive measurements was showing important variations (see Fig. 4). The initial measurements seem to indicate a faulty condition of the rotor (LSH amplitude below the line at $-45 \mathrm{~dB}$ ). However, the latter measurements led to much lower values of the LSH amplitude that correspond to healthy condition. This erratic behavior of the LSH amplitude was illustrative of the problem caused by the continuous load variation. This variation was provoking the incorrect evaluation of the LSH amplitude when the slip $s$ changed during the capture of the current. The application of ATCSA to this case (see Fig.5) shows a faulty condition of the rotor for this motor (the V-shaped pattern is present). The computation of fault severity indicators based on ATCSA leads to a value of 47.1 dB confirming the faulty condition of the rotor.

- Motor M2 is a clear case of false positive indication. The LSH amplitude, despite variable, remains in an approximate constant value after the initial measurement (see Fig. 4). However, ATCSA does not reveal any Vshaped pattern caused by the LSH (Fig. 6) and the ATCSA indicator yields a value of $69.3 \mathrm{~dB}$. This indicates that the fault is not present. The LSH in MCSA is probably caused by the oscillating nature of the load that was introducing a harmonic very similar to the LSH that was erroneously identified as the fault harmonic.

The case of Motor M3 illustrates a situation where the 

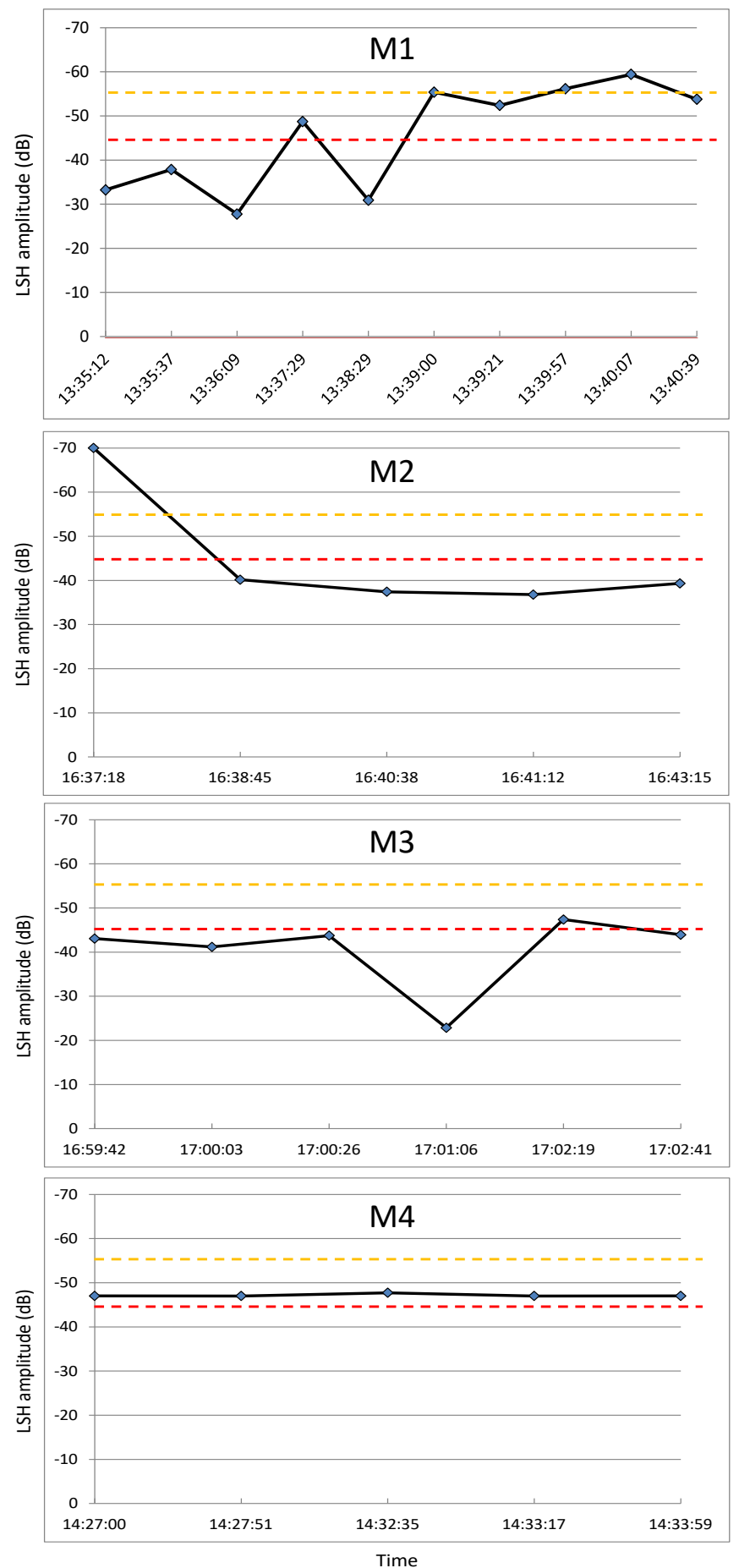

Fig. 5. Field case A: evolution of LSH for successive measurements for the four motors under study.

conclusions of MCSA and ATCSA are coincident. While the LSH amplitude, despite punctual variations, indicates a faulty condition of the rotor (Fig. 4), ATCSA reveals the presence of the pattern and yields an indicator of 39.1 dB. - Finally, the case of motor M4 is analogue to that of M2 (false positive): while the MCSA indicates faulty condition, ATCSA does not show any pattern and yields a value of $66.5 \mathrm{~dB}$, which confirms the healthy condition.
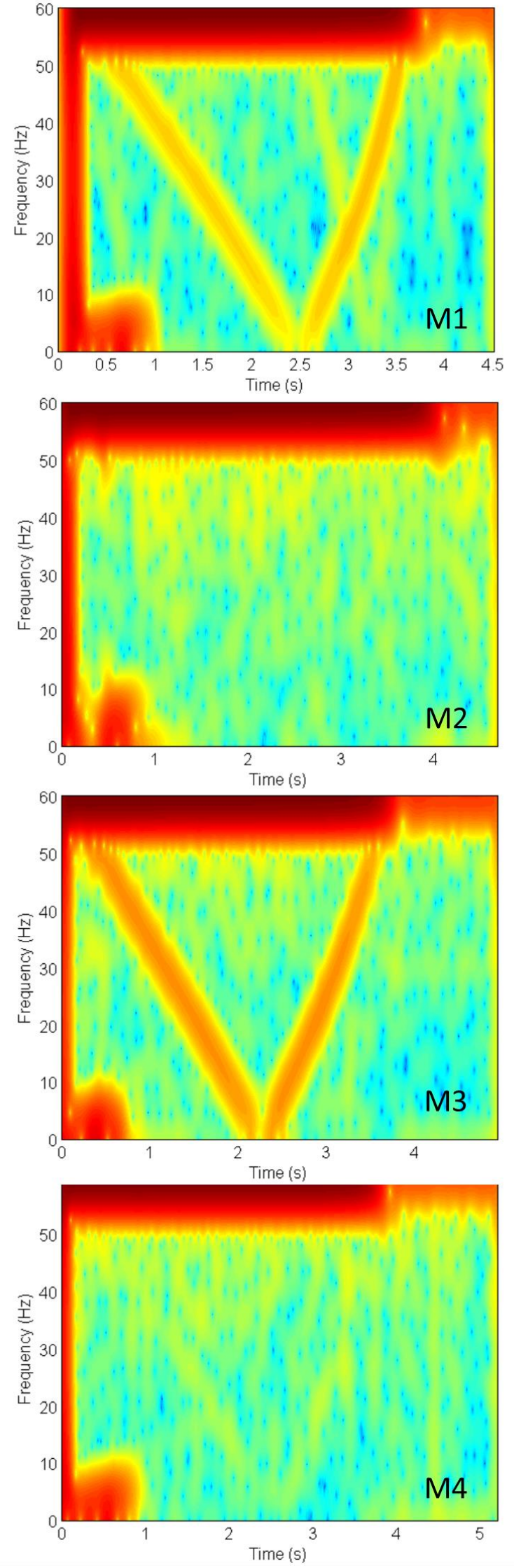

Fig. 6. Field case A: application of ATCSA to all four motors. 


\section{B. Healthy $850 / 450 \mathrm{~kW}$ motor driving a fume exhaust fan}

The diagnosed machine was a three-phase double speed $850 / 450 \mathrm{~kW}$ motor that was driving a fan for exhaust fume extraction in a mining facility in Central Europe. The motor was operating at $450 \mathrm{~kW}$ (lower speed, number of pole pairs= $5)$. The variable speed operation of this motor make it advisable to apply techniques based on the analysis of the startup current (ATCSA). Fig.7 shows the ATCSA of the startup current for this motor. It is observed how the Vshaped pattern associated with the rotor asymmetry had very low intensity. In addition, the computation of the fault severity indicator relying on the DWT led to a value of $\mathbf{5 3 . 7}$ dB (higher than the threshold level $50 \mathrm{~dB}$ ) which confirmed the healthy condition of the rotor cage.

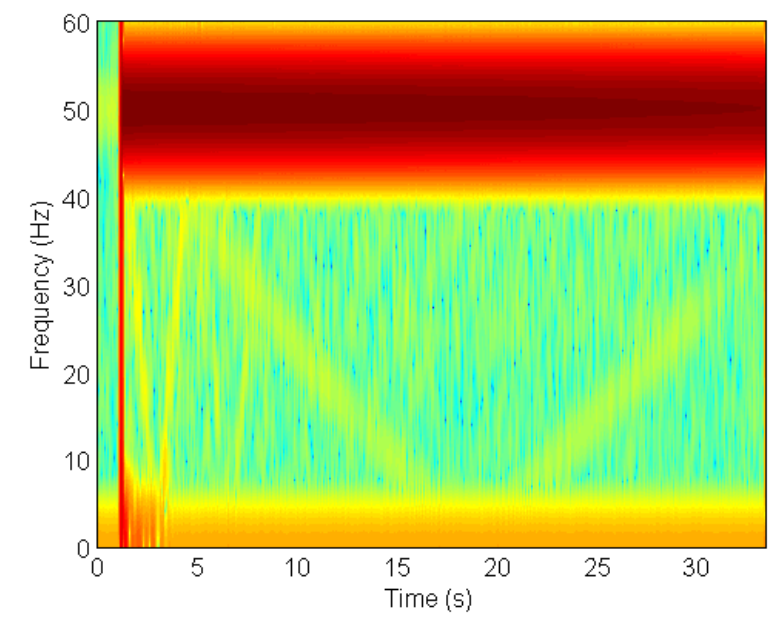

Fig. 7. Field case B: application of ATCSA to the 850/450kW motor.

\section{Faulty 850/450 kW motor driving a fume exhaust fan}

The motor diagnosed in this case was similar to the previous one, i.e., a three-phase double speed 850/450 kW motor that was driving a fan for the extraction of the exhaust fumes in a mining facility in Central Europe. Again, the motor was operating at $450 \mathrm{~kW}$ (lower speed, number of pole pairs= 5). The preliminary application of MCSA led to suspect the presence of certain damage in the rotor cage since prominent sidebands appeared at both sides of the supply frequency. In order to ratify this diagnostic and avoid a possible false indication, ATCSA was applied to the startup current.

Fig. 8 shows the ATCSA of the startup current for this motor. The V-shaped pattern associated with the rotor asymmetry is clearly observed, having a prominent intensity in the time-frequency map. In addition, the computation of the fault severity indicator based on ATCSA led to a value of 45.4 dB (lower than the threshold level) which confirmed the existence of certain damage in the rotor cage.

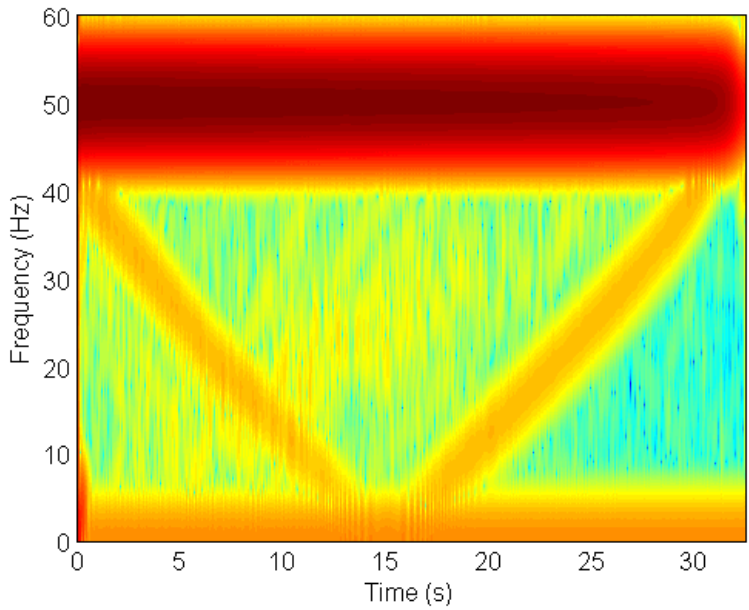

Fig. 8. Field case C: application of ATCSA to the 850/450kW motor.

\section{High speed coal mill motor with severe rotor damage}

The motor analyzed in this case was a $320 \mathrm{~kW}$ motor driving a high speed coal mill in a mining facility in central Europe. The application of the MCSA method revealed the presence of fault components with prominent amplitudes similar to those depicted in Fig.2. This indicated the possible existence of a significant asymmetry in the rotor cage. In order to ratify this diagnostic, it was decided to apply the ATCSA method. Fig.9 shows the ATCSA analysis of the startup current for this motor. Note the presence of a clear Vshaped pattern with significant intensity that is an evidence of the presence of the rotor damage. In order to determine the severity of the damage, the ATCSA-based fault severity indicator was computed, yielding a value of $\mathbf{4 2} \mathbf{~ d B}$, which was clearly indicative of a severe damage. Finally, the motor was disassembled and the rotor inspected, revealing the presence of several breakages in the rotor cage (see picture of this rotor in Fig. 1).

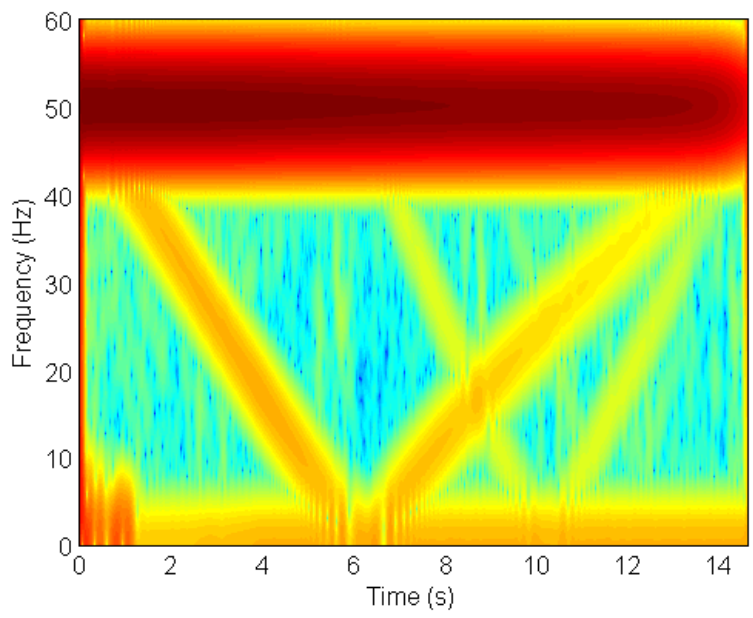

Fig. 9. Field case D: application of ATCSA to the $320 \mathrm{~kW}$ motor. 


\section{CONCLUSIONS}

In this paper, the analysis of the motor current is proposed as a valuable informational source to assess the rotor condition of induction motors operating in mining facilities. In this type of applications, the possibility of rotor damage is higher, due to the existence of frequent transient regimes caused by abrupt load variations and heavy startups that increase the thermal gradients and electrodynamic stresses in the rotor bars. The use of a simple technique able to determine the rotor condition in a fast and reliable way, without necessity of disassembling the machine becomes certainly appealing. In this context, the current monitoring technique, relying on the analysis of the waveform of the current demanded by the motor, is an excellent alternative since, in addition to its simplicity and suitability for detecting rotor damages, it enables the remote monitoring of the motor condition (the current can be measured in the motor control center, in the secondary of current transformers) in a noninvasive way.

The two main available technologies based on analysis of currents have been revised in this paper: on the one hand, the classical MCSA method, relying on the FFT analysis of the steady-state current and, on the other hand, the modern technologies based on analysis of the startup current (ATCSA). The field cases included in this paper, corresponding to motors operating in real mining facilities in different countries, prove the reliability of the joint application of both approaches. In particular, it is demonstrated how the use of ATCSA can be very useful for avoiding some drawbacks of the classical MCSA, such as its unsuitability in variable speed regimes or its occasional false indications.

With regards to the method sensitivity, in past works it was proven the effectivity of the method for detecting with accuracy severity levels of around 1 broken bar out of 28 (less than 5 percent of bars broken). The detection of the fault while it is still in such low severity levels gives enough reaction time to plan proper maintenance actions or rotor repair. Moreover, simulations indicated that under severe transients the effect of incipient faults is increased, compared to stationary conditions.

It must be remarked that the methodology can be extrapolated to the detection of other faults as well as other operation regimes, as proven in previous works [26-27]. Indeed, it is even possible to use it in inverted-fed motors. In this case the evolutions of the fault harmonics are slightly different. For instance, the LSH progressively separates from the fundamental during the startup. But, using appropriate time-frequency tools, these components can be discerned and the rotor condition diagnosed, as it was proven in previous works [26].

\section{ACKNOWLEDGMENT}

This work was supported in part by the Spanish 'Ministerio de Economía y Competitividad' (MINECO) and
FEDER program in the framework of the 'Proyectos I+D del Subprograma de Generación de Conocimiento, Programa Estatal de Fomento de la Investigación Científica y Técnica de Excelencia' (ref: DPI2014-52842-P).”

\section{REFERENCES}

[1] W.T. Thomson, M. Fenger, "Current signature analysis to detect induction motor faults" IEEE Industry Applications Magazine, July/August 2001, pp. 26-34.

[2] S.B. Lee, E. Wiedenbrug., K. Younsi, "ECCE 2013 Tutorial: Testing and Diagnostics of Induction Machines in an Industrial Environment", presented at ECCE 2013, Denver, CO, USA, Sep 2013.

[3] O.V. Thorsen and M. Dalva, "Failure Identification and Analysis for High-Voltage Induction Motors in the Petrochemical Industry," IEEE Transactions on Industry Applications, vol. 35, no. 4, pp. 810-818, July/Aug. 1999.

[4] A. Bellini, et al., "On-field experience with on-line diagnosis of large induction motors cage failures using MCSA," IEEE Trans. on Ind. Appl., pp. 1045-1053, vol. 38, no. 4, July/Aug. 2002.

[5] W.T. Thomson, I. Culbert, "Current Signature Analysis for Condition Monitoring of Cage Induction Motors”, IEEE Press, Wiley, New Jersey, 2017.

[6] J. A. Antonino-Daviu, V. Climente-Alarcón, J. Pons-Llinares and E. Wiedenbrug, "Advanced rotor assessment of motors operating under variable load conditions in mining facilities," in Proc. of the 2014 IEEE Energy Conversion Congress and Exposition (ECCE), Pittsburgh, PA, 2014, pp. 617-621.

[7] Jose A. Antonino Daviu, Joan Pons-Llinares and Sang Bin Lee, "Advanced Rotor Fault Assessment for High Voltage Induction Motors via Continuous Transforms" in Proc. of PCIC Europe, London, U.K, June 2015, pp. 57-63.

[8] J. A. Antonino-Daviu, M. Riera-Guasp, J. R. Folch, and M. Pilar Molina Palomares, "Validation of a new method for the diagnosis of rotor bar failures via wavelet transform in industrial induction machines," IEEE Trans. Ind. Appl., vol. 42, pp. 990-996, 2006.

[9] Jose Antonino-Daviu, Martin Riera-Guasp, Joan Pons-Llinares, Jongbin Park, Sang Bin Lee, Jiyoon Yoo and Christian Kral, "Detection of Broken Outer Cage Bars for Double Cage Induction Motors under the Startup Transient", IEEE Transactions on Industry Applications, vol. 48, no.5, pp. 1539-1548, Sept-Oct. 2012.

[10] J. A. Antonino-Daviu, S. B. Lee and E. Wiedenbrug, "Reliable detection of rotor bar failures in induction motors operating in petrochemical plants," in Proc. of the 2014 Petroleum and Chemical Industry Conference Europe (PCIC Europe), Amsterdam, 2014, pp.19.

[11] C. Yang, T-J. Kang, D. Hyun, S. Lee, J. Antonino-Daviu, J. PonsLlinares, "Reliable Detection of Induction Motor Rotor Faults Under the Rotor Axial Air Duct Influence," IEEE Trans. Ind. Appl., vol. 50, no. 4, pp. 2493-2502, Jul.-Aug. 2014.

[12] Jose A . Antonino-Daviu, A.Quijano-Lopez, V. Fuster-Roig and C. Nevot, "Case Stories on Induction Motors Fault Diagnosis based on Current Analysis", in Proc. of PCIC Europe 2016, Berlin, Germany, June 2016, pp. 115-123.

[13] J. Pons-Llinares, J. A. Antonino-Daviu, M. Riera-Guasp, S. Bin Lee, T. J. Kang and C. Yang, "Advanced Induction Motor Rotor Fault Diagnosis Via Continuous and Discrete Time-Frequency Tools," IEEE Transactions on Industrial Electronics, vol. 62, no. 3, pp. 17911802, March 2015.

[14] J. Antonino-Daviu, A. Q. López, M. Rubbiolo and V. ClimenteAlarcon, "Diagnosis of the rotor condition in electric motors operating in mining facilities through the analysis of motor currents," 2017 IEEE Industry Applications Society Annual Meeting, Cincinnati, OH, 2017, pp. 1-8.

[15] I.M. Culbert, and W. Rhodes, "Using current signature analysis technology to reliably detect cage winding defects in squirrel-cage induction motors," IEEE Trans. on Ind. Appl., vol. 43, no.2, pp.422428, Mar./Apr. 2007. 
[16] Manés F. Cabanas, Manuel G. Melero, Gonzalo A. Orcajo, José M. Cano, Juan Solares, Maintenance and Diagnostic Techniques for Rotating Electric Machinery, Marcombo Boixareu, 1999.

[17] R. R. Schoen and T.G. Habetler. "Evaluation and Implementation of a System to Eliminate Arbitrary Load Effects in Current-Based Monitoring of Induction Machines," IEEE Trans. Ind. Appl., vol.33, no. 6, pp. 1571-1577, November/December 1997.

[18] S. Shin, J. Kim, S. B. Lee, C. Lim and E. J. Wiedenbrug, "Evaluation of the Influence of Rotor Magnetic Anisotropy on Condition Monitoring of Two-Pole Induction Motors," IEEE Transactions on Industry Applications, vol. 51, no. 4, pp. 2896-2904, July-Aug. 2015

[19] H. Kim, S. B. Lee, S. Park, S. H. Kia and G. A. Capolino, "Reliable Detection of Rotor Faults Under the Influence of Low-Frequency Load Torque Oscillations for Applications With Speed Reduction Couplings," in IEEE Transactions on Industry Applications, vol. 52, no. 2, pp. 1460-1468, March-April 2016.

[20] M. Riera-Guasp, J.A. Antonino-Daviu, G. A. Capolino, "Advances in Electrical Machine, Power Electronic, and Drive Condition Monitoring and Fault Detection: State of the Art," IEEE Transactions on Industrial Electronics, vol.62, no.3, pp. 1746-1759, Mar. 2015.

[21] Aurelien Prudhom, Jose Antonino-Daviu, Hubert Razik, Vicente Climente-Alarcon, "Time-frequency vibration analysis for the detection of motor damages caused by bearing currents", Mechanical Systems and Signal Processing, Volume 84, Part A, 1 February 2017, Pages 747-762.

[22] L. Cohen, Time-Frequency Analysis. A.V. Oppenheim, Ed. Prentice Hall Signal Processing Series, New Jersey, 1995.

[23] C.S. Burrus, R.A. Gopinath and H. Guo, Introduction to Wavelets and Wavelet Transforms a primer, Prentice Hall (1998)

[24] S. Mallat, A Wavelet Tour of Signal Processing. New York: Academic Press, (1999).

[25] V. Climente-Alarcon, D. Nair, R. Sundaria, J. A. Antonino-Daviu, A. Arkkio, "Combined model for simulating the effect of transients on a damaged rotor cage," IEEE Trans. Ind. Appl., Vol. 53, nº. 4, pp. 35283537, Jul.-Ago. 2017.

[26] J. Pons-Llinares, D. Morinigo-Sotelo, O. Duque-Perez, J. AntoninoDaviu and M. Perez-Alonso, "Transient detection of close components through the chirplet transform: Rotor faults in inverter-fed induction motors," IECON 2014 - 40th Annual Conference of the IEEE Industrial Electronics Society, Dallas, TX, 2014, pp. 3386-3392.

[27] J. Pons-Llinares, J. Antonino-Daviu, J. Roger-Folch, D. MoríñigoSotelo, O. Duque-Pérez, "Mixed eccentricity diagnosis in Inverter-Fed Induction Motors via the Adaptive Slope Transform of transient stator currents," Mechanical Systems and Signal Processing, vol. 48, no.1-2, pp. 426-435, Oct. 2014

\section{BIOGRAPHIES}

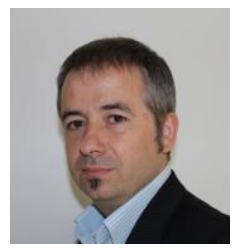

Jose A. Antonino-Daviu (S'04, M'08, SM'12) received his M.S. and $\mathrm{Ph}$. D. degrees in Electrical Engineering, both from the Universitat Politècnica de València, in 2000 and 2006, respectively. He also received his Bs. in Business Administration from Universitat de Valencia in 2012. He was working for IBM during 2 years, being involved in several international projects. Currently, he is Associate Professor in the Department of Electrical Engineering of the mentioned University, where he develops his docent and research work. He has been invited professor in Helsinki University of Technology (Finland) in 2005 and 2007, Michigan State University (USA) in 2010, Korea University (Korea) in 2014 and Université Claude Bernard Lyon 1 (France) in 2015. $\mathrm{He}$ is IEEE Senior Member since 2012 and he has published over 160 contributions, including international journals, conferences and books. He is also Associate Editor of IEEE transactions on Industrial Informatics and has been Guest Editor in IEEE transactions on Industrial Electronics. He was General Co-Chair of IEEE SDEMPED 2013

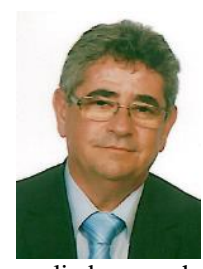

Alfredo Quijano-Lopez was born in 1960 in Valencia, Spain. He received the Electrical Engineer degree and the $\mathrm{Ph} . \mathrm{D}$. degree from Universitat Politècnica de València, in 1986 and 1992, respectively. He is the Head of the Instituto Tecnológico de la Energía. He is also a professor and a researcher at the Universitat Politècnica de València in the Electrical Engineering Department. His current research activity is focused on applied research for the Energy area and the electrical technology including renewable energies, high voltage, metrology, new materials and applications and research results transfer to companies.

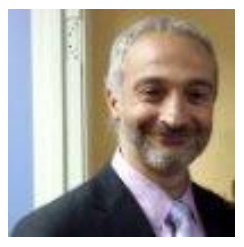

Martin Rubbiolo received his M.S. in Electrical Engineering from the University of Colorado, Boulder Campus, CO, USA. He worked for 9 years as Head of Sales for Latin America for the former company Baker Instrument Company, SKF today. During that time he worked as a trainer, organizing and teaching courses within the US and in Latin America. Ing. Rubbiolo has extensive experience in the area of electrical tests Megohm, IP, Surge, Impulse and HiPot.

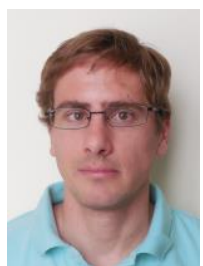

Vicente Climente-Alarcon (S'11-M'12), received the M.Sc. degrees in Chemical and Industrial Engineering in 2000 and 2011, and the Ph.D. degree in Electrical Engineering in 2012, all from the Universitat Politècnica de València (Spain). He worked as Adjunct Professor in the School of Industrial Engineering of the mentioned university, on research tasks in the area of condition monitoring of electrical machines and externally as a consultant in automation and management of power systems. In 2013 and from 2014 to 2017 he was with the Department of Electrical Engineering and Automation, Aalto University, Espoo, Finland, where he was involved in research in the area of multiphysics simulations as visiting scholar, Postdoctoral Researcher and Docent. Currently he is a Research Associate at the Department of Materials Science and Metallurgy, University of Cambridge, Cambridge, UK, working on technological applications of superconductivity. 